\title{
Frontières
}

\section{Du culte des morts au combat politique}

Paris, 1814-1840

\section{Emmanuel Fureix}

Volume 19, numéro 1, automne 2006

Enjeux politiques et mort

URI : https://id.erudit.org/iderudit/016630ar

DOI : https://doi.org/10.7202/016630ar

Aller au sommaire du numéro

Éditeur(s)

Université du Québec à Montréal

ISSN

1180-3479 (imprimé)

1916-0976 (numérique)

Découvrir la revue

Citer cet article

Fureix, E. (2006). Du culte des morts au combat politique : paris, 1814-1840.

Frontières, 19(1), 15-20. https://doi.org/10.7202/016630ar
Résumé de l'article

Des débuts de la Restauration au retour des Cendres de Napoléon (décembre 1840), le culte des morts en vient à incarner les luttes politiques de la France postrévolutionnaire. Culte des victimes de la Révolution, funérailles dynastiques, funérailles nationales, funérailles d'opposants politiques participent à l'apprentissage de la politique moderne. Le Paris de l'âge romantique est le théâtre de cette politisation du culte des morts. L'article étudie trois aspects singuliers de ce processus : le rôle des émotions et du sentiment, la redéfinition du sacré en politique et l'émergence d'une prise de parole contestataire.
Ce document est protégé par la loi sur le droit d'auteur. L'utilisation des services d'Érudit (y compris la reproduction) est assujettie à sa politique d'utilisation que vous pouvez consulter en ligne.

https://apropos.erudit.org/fr/usagers/politique-dutilisation/ 


\section{Résumé}

Des débuts de la Restauration au retour des Cendres de Napoléon (décembre 1840), le culte des morts en vient à incarner les luttes politiques de la France postrévolutionnaire. Culte des victimes de la Révolution, funérailles dynastiques, funérailles nationales, funérailles $d$ 'opposants politiques participent à l'apprentissage de la politique moderne. Le Paris de l'âge romantique est le théâtre de cette politisation du culte des morts. L'article étudie trois aspects singuliers de ce processus: le rôle des émotions et du sentiment, la redéfinition du sacré en politique et l'émergence d'une prise de parole contestataire.

Mots clés: histoire de la mort enterrements - histoire politique Paris à l'âge romantique - émotions en politique.

\begin{abstract}
From the early beginnings of the Restoration to the return of the ashes of Napoleon (December 1840), the cult of the dead exemplified the political struggles of post-revolutionary France. The cult of the victims of the Revolution, dynastic funerals, national funerals, funerals of political opponents, all play their part in forming our modern politics. Paris was a theatre for this politicization of the cult of the dead during the Romantic period. This papers analyzes three singular aspects of this process: the role of emotions and sentiment, the re-definition of sacred in politics, and the emergence of public protest.
\end{abstract}

Keywords: history of death - burial political history - Paris Romantic period - emotions in politics.

\section{DU CULTE DES MORTS AU COMBAT POLITIQUE Paris, 1814-1840}

\section{Emmanuel Fureix, \\ maître de conférences en histoire contemporaine, Université Paris XII, Institut Jean-Baptiste-Say.}

La mort et sa ritualité se prêtent à maints usages politiques: mise en scène de la souveraineté à travers des funérailles d'État, inscription de la légitimité et de la continuité du pouvoir, fabrication imaginaire d'un ordre sociopolitique, inculcation de sentiments obligés lors de deuils officiels, construction d'une mémoire étatique ou subversive autour de "grands ancêtres", simple manifestation d'une opinion ou d'une croyance au cours d'enterrementsmanifs... Depuis une trentaine d'années, des terrains d'enquête très variés, tant en histoire (Giesey, 1987; Julliard, 1999; Tartakowsky, 1999; Ben Amos, 2000) qu'en anthropologie ou en science politique, ont permis d'affiner chacune de ces hypothèses, sur la longue durée comme sur le temps court (de Baecque, 1997), voire très court (Dewerpe, 2006). De riches débats historiographiques en ont découlé, en particulier autour de la performativité du rituel funèbre (Boureau, 1988), tandis que le regain d'intérêt récent pour les émotions en histoire (Reddy, 2001) permet de renouveler encore les approches du deuil en politique.

De cette constellation d'enquêtes, notre recherche (Fureix, 2003) vise à la fois à faire converger les questionnements et à concentrer l'attention sur une période (l'âge romantique - ici entre 1814 et 1840 , du début de la Restauration des Bourbons au retour des cendres de Napoléon) et une ville (Paris), où le culte des morts entre de plain-pied en politique et façonne les cultures politiques. Alors que se poursuit l'éloignement des vivants et des morts entamé partout en Occident à la fin du $\mathrm{XVIII}^{\mathrm{e}}$ siècle, jamais sans doute les morts n'ont été autant sollicités par les vivants pour légitimer, façonner, dire le politique. Jamais, pour paraphraser Maurice Barrès, la «vertu sociale d'un cadavre ${ }^{1}$ n'a été autant exploitée. La révolution funéraire apparue au tournant du siècle - fantasme d'une mort pacifiée, hygiéniste et régénératrice (Hintermeyer, 1981), réaffirmation de la dignité du cadavre, de son individualité, obsession de la trace dans une "société de conservation » (Urbain, 1978), construction de grandes nécropoles modernes (Lassere, 1997a) - a permis aux passions politiques du moment de s'incarner de manière originale. En outre, la mémoire proche de la Révolution et de ses victimes outragées jusque dans leur sépulture a réactivé des rites funéraires oubliés. Entre 1814 et 1840, hommages expiatoires aux victimes royales de la Révolution, funérailles dynastiques de la Restauration, funérailles nationales sous la monarchie de Juillet, enterrements d'opposants notoires, deuil à distance puis funérailles officielles de Napoléon, hommages aux victimes des pouvoirs, insurgés ou condamnés à morts politiques, se succèdent et se répondent, s'adaptent à l'âge du « gouvernement représentatif » (cf. tableau I). Des 
panthéons rivaux, des rituels divergents, des mémoires antagoniques entrent au service du politique, entendu, à la manière de Carl Schmitt, comme «discrimination de l'ami et de l'ennemi ${ }^{2}$ ».

Nous voudrions ici esquisser les contours de ce qui apparaît à l'âge romantique comme un modèle parisien de la mort en politique: l'apprentissage de la représentation politique par le culte des morts. Trois voies principales permettent d'éclairer cet apprentissage politique : le rôle du sentiment en politique, la construction d'une sacralité politique autour du culte des morts, l'émergence d'une prise de parole protestataire par les hommages rendus aux défunts.

\section{LE POUVOIR DES LARMES: LES ÉMOTIONS DU DEUIL \\ EN POLITIQUE}

On ne comprendrait pas, sans un détour par les émotions et le sentiment, le rôle du deuil en politique à l'âge romantique. Les hommes du XIX ${ }^{\mathrm{e}}$ siècle, qu'ils soient royalistes intransigeants, doctrinaires ou libéraux, dénoncent l'usage intempérant des passions en politique. La Terreur de 17931794 et le désordre des passions, l'effroi comme instrument de domestication politique (Wahnich, 2002), apparaissent comme des contre-modèles. L'émotion elle-même, encore largement confondue dans le lexique politique avec l'émeute ${ }^{3}$, et par conséquent avec des passions actualisées en violences, est discréditée. Mais l'abstraction de la raison et la négation absolue des affects sont tout autant décriées par les partisans du régime des Bourbons, comme des héritages pervers de la Révolution.

À leurs yeux, seul le sentiment, entendu comme un transport affectif inscrit dans la durée et spiritualisé, à la différence du sensualisme des Lumières, peut rétablir une communauté politique brisée par l'expérience de la Révolution et de l'Empire. C'est l'opinion d'un royaliste modéré comme Maine de Biran, pour lequel aucune croyance, politique comme religieuse, ne saurait perdurer sans sentiment, qui seul peut «devenir un principe d'action pour les peuples comme pour les individus ${ }^{4} »:$ «sans le sentiment monarchique, sans le respect et l'amour pour le roi légitime, il ne peut y avoir pour la France ni religion, morale, ni patrie. Tous les liens sociaux viennent se rattacher à ce sentiment ${ }^{5} »$. Le sentiment agit en modérateur des passions politiques, à condition qu'il soit maîtrisé. Ce retour au sentiment et à la sensibilité comme fondement du lien social et politique est indissociable d'une spiritualité néocatholique du dolorisme et des larmes, dont le Chateaubriand du Génie du christianisme (1802) et le Ballanche Du sentiment (1801) se sont faits les hérauts. Indissociable également des émotions esthétiques et des souffrances intériorisées de la nouvelle génération romantique (Vincent-Buffault, 1986 , p. 136), et de la valorisation médicale des larmes sensibles (article «larmes» du Dictionnaire des sciences médicales de Panckoucke, 1818).

Pour toutes ces raisons, le deuil et les larmes sont exaltés en politique, au premier chef par les partisans des Bourbons. De

\section{Tableau I - REPĖRES CHRONOLOGIQUES SUR LES FUNÉRAILLES POLITIQUES DANS LA CAPITALE (1814-1840)}

\section{Principales funérailles d’État}

- 21 janvier 1815: exhumation et funérailles de Louis XVI et Marie-Antoinette (Saint-Denis)

- 21 mars 1816: exhumation et funérailles du duc d'Enghien (Vincennes)

- janvier 1817: exhumation et réinhumation des rois, reines, princes et princesses "profanés" en 1793 à Saint-Denis

- 26 mai 1818: funérailles du prince de Condé (Saint-Denis)

- 13 février / 14 mars 1820: assassinat et funérailles du duc de Berry (Saint-Denis)

- 16 septembre / 25 octobre 1824: mort et funérailles de Louis XVIII (Saint-Denis)

- 27 août / 4 septembre 1830: mort et funérailles du duc de Bourbon

- 19 mai 1832: funérailles nationales de Casimir Perier, président du Conseil des ministres

- 28 juillet / 5 août 1835: attentat de Fieschi contre Louis-Philippe et funérailles des victimes aux Invalides

- 5 décembre 1837: funérailles du général Damrémont aux Invalides

- 15 décembre 1840: funérailles de Napoléon (mort le 5 mai 1821) aux Invalides

\section{Principales funérailles d'opposition}

- 6 juin 1820: funérailles de l'étudiant Lallemand

- 4 juin 1823: funérailles du maréchal Davout

- 30 novembre 1825 : funérailles du général Foy (député libéral)

- 20 octobre 1826: funérailles civiles de l'acteur Talma

- 30 mars 1827: funérailles du duc de La Rochefoucault-Liancourt

- 24 août 1827: funérailles civiles du député Manuel

- 12 décembre 1830: funérailles de Benjamin Constant

- 16 mai 1831 : funérailles du député libéral Labbey de Pompières

- 30 mai 1831 : funérailles de l'abbé Grégoire

- 5 juin 1832: funérailles civiles du général Lamarque, prélude à une émeute républicaine

- 1 er février 1834: funérailles civiles du jeune député Dulong, mort en duel

- 22 mai 1834: funérailles du général La Fayette

- 22 juin 1836: funérailles de l'abbé Sieyès

- 25 juillet 1836: funérailles du républicain Armand Carrel, mort en duel 


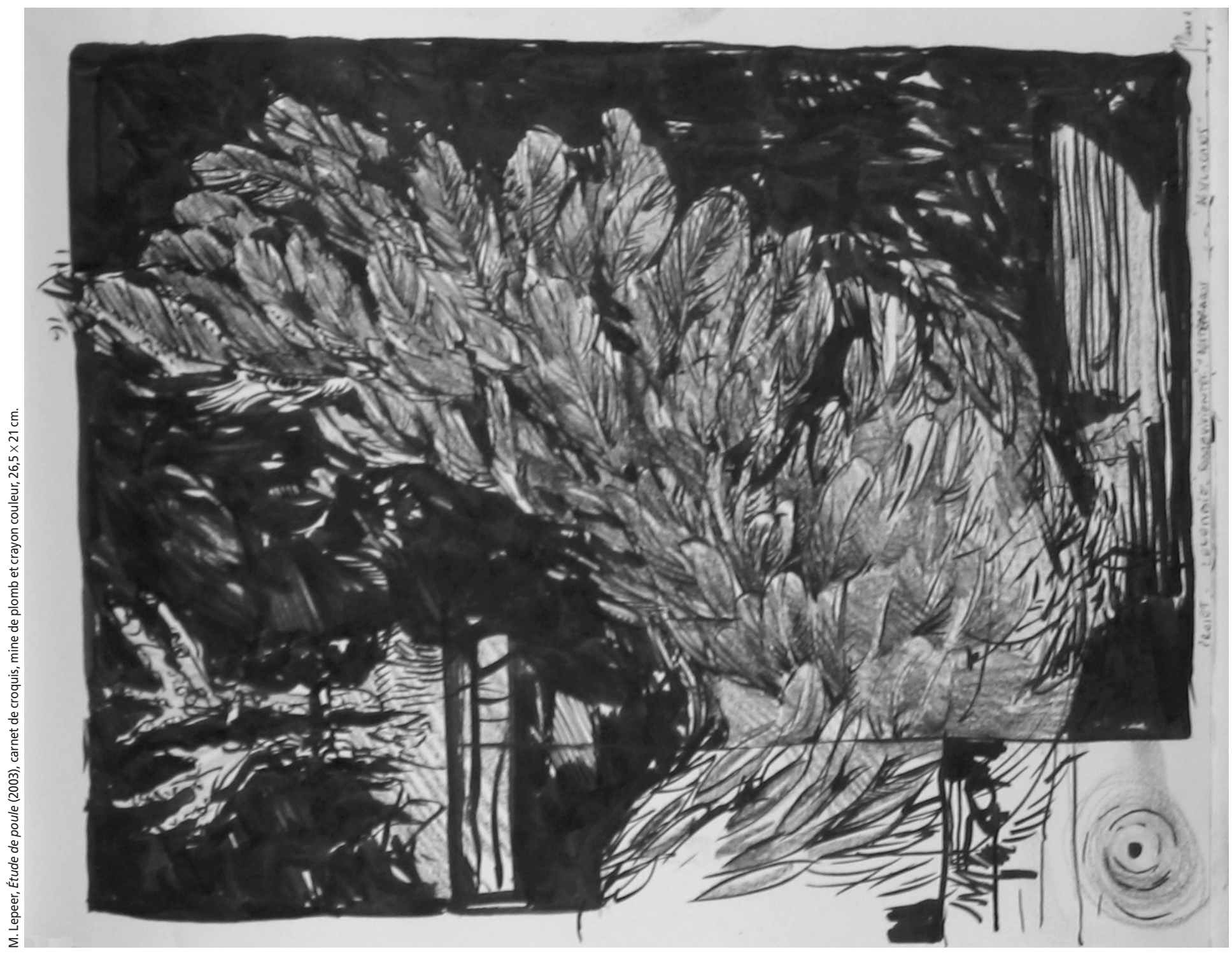

même que la douleur se révèle rédemptrice en matière spirituelle, de même le deuil devient fondateur en matière politique. Le partage d'émotions morales rapproche les individus autour de leur souverain. L'expérience tragique du régicide du 21 janvier 1793 peut-être retournée en célébration expiatoire, utile exercice de repentir national pour les "crimes de la Révolution». Les ultraroyalistes, de même que le clergé, insistent tout particulièrement sur cette fonction expiatoire du 21 janvier, journée de deuil national imposée par la loi du 19 janvier 1816 (Fureix, 2005). Aux yeux des autorités compte surtout la fusion de la communauté dans l'échange de larmes. À en croire les rapports de police sur ces cérémonies, fort stéréotypés il est vrai, la contagion des larmes est toujours irrépressible lorsque est lu en chaire le «testament de Louis XVI » rédigé à la tour du Temple en décembre 1792, qui rappelle les souffrances du « roi-martyr » et offre à tous la leçon du pardon aux ennemis politiques.
Quant aux libéraux, s'ils fondent d'abord la délibération politique sur le terrain de la raison individuelle (Reddy, 2001, p. 217), ils ne repoussent pas pour autant tout usage politique des sentiments. Si la «liberté des modernes », selon les termes de Benjamin Constant, repose sur la jouissance privée des sentiments, et non leur imposition par l'État, reste qu'elle n'exclut pas le partage public des affections. Le deuil collectif, chez les libéraux et les républicains des années 1820 et 1830 , est perçu comme un moyen d'affirmation de la nation souveraine. Lors des grands enterrements-manifestations des années 1820-1830, celui du général Foy (1825), de Manuel (1827), de Constant (1830), de Lamarque (1832), de La Fayette (1834), les émotions partagées définissent les contours d'une communauté politique opposante. À la différence du dolorisme bourbonien, les larmes ne sont pas seules convoquées: applaudissement, cris, vivats sont tout aussi présents au sein des cortèges. Le mélange des émotions du deuil oriente en effet la commémoration du défunt vers un avenir collectif, fait de progrès et d'espérance patriotique. Ainsi Edgar Quinet, après avoir assisté aux funérailles du ténor libéral Foy, en 1825, peut-il écrire à sa mère :

«J'ai voulu t'écrire, il y a plusieurs jours, le lendemain des funérailles du général Foy. Rien n'effacera ce jour de mon souvenir, et j'ai conçu d'immenses espérances, et une joie religieuse de tout ce qui a alors frappé mes yeux. C'est une douce pensée que le pays quand on le voit renaître de ses cendres; $[. .$.$] ce mot de France com-$ mence à réveiller une foule d'émotions nationales qui semblaient être éteintes pour jamais ${ }^{6}$. »

Les émotions du deuil en politique ne se réduisent pas à l'affliction. Le glissement du sentiment aux passions politiques est possible, lorsque est convoquée une mémoire sanglante et que se diffusent la peur et 
l'effroi. Les funérailles républicaines des années 1830 l'attestent pleinement. Outre les habituels vivats et cris de célébration, pointent parfois des cris de menace, qui réintroduisent au cœur du deuil la pratique révolutionnaire du serment, agrémenté d'appels à la vengeance. Ainsi un membre de la Société des Amis du peuple, lors des funérailles de Benjamin Constant, harangue-t-il en ces termes la foule en deuil:

«Amis du peuple, ennemis de l'aristocratie, ennemis des privilèges, les serments renouvelés chez les morts ont quelque chose de terrible: jurons ici, jurons que nos journées de Juillet, si chèrement achetées de la vie de nos frères, ne seront pas perdues. C'est à nous d'honorer un sang si pur en obéissant aux vœux des mourants qui l'ont répandu; les taches en fument encore: ils ont fait plus que leur devoir: faisons le nôtre ${ }^{7}$.»

\section{UNE RELECTURE DU SACRÉ EN POLITIQUE}

Entre la Restauration et la monarchie de Juillet, le culte des morts contribue aussi à redéfinir une sacralité politique, mise à mal dans sa version monarchique par l'expérience de la Révolution. La sacralité traditionnelle de la royauté, celle du tremendum, de la fureur et du mystère (Otto, 1929), déjà ébranlée par le processus de désacralisation entamé au XVIII ${ }^{\mathrm{e}}$ siècle, ne pouvait être rétablie comme telle. Le régicide de 1793 , qui visait non seulement la personne de Louis XVI, mais aussi le corps mystique et politique de la monarchie, l'avait définitivement éradiquée. Mais le sacré, précisément à partir du régicide lui-même, pouvait être ressourcé par le martyr, l' "holocauste» d'un roi, qui, à entendre les oraisons funèbres prononcées en 1815 à l'occasion de l'anniversaire du 21 Janvier, se serait offert pour le salut de l'humanité. À l'issue de son procès, Louis XVI serait devenu «agneau de Dieu», l'échafaud de la place de la Concorde un nouveau «Calvaire», le rapport du roi défunt avec ses sujets une «nouvelle alliance». Louis XVI inaugure ainsi la série des «Christ romantiques » étudiés naguère par Frank Paul Bowman (1973).

L'exhumation des corps de Louis XVI et Marie-Antoinette, suivie de leur transfert à Saint-Denis le 21 janvier 1815, s'apparente à cet égard à une véritable "invention de reliques ». Le cadavre de Louis XVI, «identifié» par son emplacement, réduit aux «ossements d'un corps d'homme, dont plusieurs, presque entièrement corrodés, étaient prêts de tomber en poussière ${ }^{8}$ », devient le support d'un culte qui vise à réactiver la « religion royale». Louis XVI, imprudemment présenté comme «Saint
Louis II » sous la Restauration, devient le roi tutélaire du régime, aux côtés de Louis IX et de Henri IV. La cause de béatification, introduite par la duchesse d'Angoulême, fille du "roi martyr», échoue cependant en 1820 auprès de la Congrégation des rites à Rome (Boutry, 1990).

Reste que la Restauration fonde bel et bien une monarchie de type sacrificielle, ancrée dans le culte de ses martyrs politiques, dans le rappel expiatoire des outrages subis sous la Révolution. C'est en ce sens qu'il faut comprendre le goût prononcé du régime pour les exhumations publiques, celle de Louis XVI et Marie-Antoinette, déjà évoquée, mais aussi celle du duc d'Enghien, fusillé dans les fossés du château Vincennes en 1804, celle des Capétiens, Valois et Bourbons que les révolutionnaires avaient chassés de leurs tombeaux en 1793 et rassemblés dans une fosse commune près de la basilique de Saint-Denis, mais aussi celle des tantes de Louis XVI, mortes en exil à Trieste et inhumées à Saint-Denis en 1817. Il s'agit, dans le domaine funéraire, de «renouer la chaîne des temps» de la monarchie, mais aussi d'assimiler les restes corrompus des princes aux reliques d'une dynastie souffrante. La mémoire du régicide et des profanations de cadavres de 1793 vise à restaurer l'interdit absolu du sacrilège et à garantir ainsi l'inviolabilité de la famille royale. Autour d'un culte funèbre à tonalité expiatoire se définissent les limites d'un tabou, sans lequel il n'est pas de sacré selon Durkheim (1908), les « choses sacrées sont celles que les interdits protègent et isolent» du monde profane. La répétition du régicide sous la forme de l'assassinat politique du duc de Berry, espoir de la dynastie, le 13 février 1820, n'invalide pas cette mise en scène. La mort douloureuse, mais apaisée du neveu de Louis XVIII, accentue le désir d'expiation et semble attester un pacte dynastique conclu entre les Bourbons et la «belle mort » chrétienne et magnanime. Le duc de Berry, comme son oncle Louis XVI, a pardonné à son assassin. La Restauration rétablit donc, grâce au culte des morts, une sacralité à forte dose de religiosité, même si elle ne s'accompagne pas de croyances spécifiques - ainsi le corps de «Saint Louis II » ne fait-il pas l'objet de croyances thaumaturgiques...

La monarchie de Juillet repose sur un régime de sacralité différent, éclectique, et d'un moindre degré de religiosité. La personne du roi demeure, aux termes de la Charte constitutionnelle de 1830, inviolable et sacrée. Mais cette sacralité ne dérive pas d'une investiture divine ni même providentielle, mais d'une nation abstraite, distincte du peuple auquel la souveraineté n'est pas reconnue. Ainsi le culte des morts de la révolution de juillet 1830 est-il placé au cœur de la mise en scène du pouvoir. L'une des deux fêtes nationales régulières du régime de Louis-Philippe, l'anniversaire des journées de 1830 , est centrée sur la célébration funèbre des «braves de Juillet» (officiellement environ cinq cents, dont les noms seront inscrits à partir de 1840 sur la colonne de Juillet, place de la Bastille). Ces martyrs politiques, issus pour l'essentiel du monde de l'échoppe et de la boutique, voient toutefois leurs origines sociales occultées dans une abstraction volontaire. Autre matrice de la sacralité orléaniste, le culte éclectique des "grands hommes " emprunte prudemment à la Révolution française. Le Panthéon est rétabli dans sa fonction civique (Ozouf, 1984) par une ordonnance du 26 août 1830 ; un projet de loi sur les conditions de panthéonisation est débattu à la Chambre des députés en décembre 1830, sans aboutir pour autant, faute de consensus sur la définition $\mathrm{du}$ "grand homme». Une panthéonisation des morts de juillet 1830 est un temps évoquée, avant d'être répudiée pour inconvenance politique - n'était-ce pas là sacraliser le droit à l'insurrection associée à la Constitution montagnarde de 1793 ? En revanche, Louis-Philippe eut à cœur d'honorer les morts illustres par des hommages capables de récapituler l'histoire nationale dans ses traditions multiples, éventuellement conflictuelles. Ainsi le régime orléaniste réussit-il l'exploit de mettre en scène tout à la fois les funérailles du duc de Bourbon, issu de la branche aînée, du président du Conseil Casimir Perier, incarnation du nouvel «ordre bourgeois ", des victimes de l'attentat de Fieschi en 1835, érigés en martyrs d'un complot républicain, et de Napoléon en 1840, devenu figure tutélaire de la Grande Nation. Avec la monarchie de Juillet, on passe ainsi d'un culte des morts de tradition dynastique à l'invention, encore timide, des «funérailles nationales» que la Troisième République saura mettre à l'honneur (Ben Amos, 2000).

Le culte des morts entre au service d'un régime de sacralité complexe, plus éclectique et plus laïc que sous la Restauration, au risque de voir les opposants républicains et bonapartistes s'approprier des célébrations demeurées abstraites à force de prudence. Ainsi le retour des cendres de Napoléon $1^{\mathrm{er}}$ s'accompagna-t-il de gestes séditieux: le long du cortège funèbre, les cris de «À bas Guizot!", «Haîne aux traîtres!», « À bas les traités de 1815!» se sont fait entendre. De même, lors du transfert des cendres des insurgés de 1830 sous la colonne de Juillet, le 28 juillet 1840 , des ouvriers et étudiants entonnèrent $L a$ Marseillaise, hymne alors séditieux. 


\section{UNE PRISE DE PAROLE DÉTOURNÉE?}

De fait, autour des cultes funèbres se déploie une prise de parole contestataire, qui contribue au renouvellement du répertoire d'action collective repéré par Charles Tilly (1984). Une trentaine d'enterrements d'opposition eurent ainsi lieu à Paris dans les années 1820 et 1830, rassemblant parfois des foules imposantes - près de 100000 personnes aux funérailles de Manuel en 1827 et de Benjamin Constant en 1830. Cette gestuelle d'abord libérale, puis républicaine constitue un maillon décisif et longtemps négligé dans la genèse de la manifestation contemporaine (Fureix, 2002 et 2004). Elle rendit possible, dans un moment de peur de la foule dans l'espace public, une manifestation relativement paisible et ritualisée de l'opinion publique dans les rues et cimetières de Paris. Le "tribunal de l'opinion", né au XVIIIe siècle, trouva une incarnation nouvelle dans la mort, pour des raisons qui tiennent tout à la fois aux conditions politiques des monarchies censitaires et aux nouvelles sensibilités funéraires apparues au tournant du siècle.

Victor Hugo dans les Misérables, se passe par la colonne Vendôme, lieu de mémoire patriotique et bonapartiste, avant qu'il ne marque une station place de la Bastille, et que des républicains ne tentent un transfert manqué vers le Panthéon... De même, les couronnes funèbres qui se généralisent dans les cimetières de l'âge romantique prennent un sens particulier lors des funérailles opposantes: ces couronnes d'immortelles, mais aussi de laurier et de chêne, deviennent des couronnes civiques d'hommage au "grand homme», par analogie avec la Révolution française. Au cours de la monarchie de Juillet s'ajoutent des bannières corporatives - étudiantes et ouvrières -, nationales, des exilés étrangers ou proprement politiques - le drapeau rouge lors des funérailles de Lamarque - qui politisent plus ostensiblement les cortèges funèbres. Par ailleurs, les grands cimetières nés du célèbre décret napoléonien de prairial, an XII (Bertrand, 1988), du Père-Lachaise (1804), de Montparnasse (1824) et de Montmartre (1825), deviennent le centre de souscriptions funéraires et de pèlerinages proprement politiques.

\section{LES ÉMOTIONS DU DEUIL EN POLITIQUE}

NE SE RÉDUISENT PAS À L'AFFLICTION.

\section{LE GLISSEMENT DU SENTIMENT AUX PASSIONS POLITIQUES}

\section{EST POSSIBLE, LORSQUE EST CONVOQUÉE UNE MÉMOIRE SANGLANTE}

ET QUE SE DIFFUSENT LA PEUR ET L'EFFROI.

C'est sur ce dernier point que nous voudrions insister. Les gestes de protestation ou de contestation qui se nichent au cœur de ces enterrements libéraux, puis républicains, résultent en effet de déplacements politiques de rites funéraires nouveaux. Le processus est analogue au bricolage symbolique ou «keying» décrit par Erving Goffman (Goffmann, 1991, p. 52) et qu'il définit comme "un ensemble de conventions par lequel une activité donnée, déjà pourvue de sens par l'application d'un cadre primaire, se transforme en une autre activité qui prend la première pour modèle mais que les participants considèrent comme sensiblement différente». Il en est ainsi du cortège d'accompagnement du défunt de la maison mortuaire vers l'église puis le cimetière extra-muros - le plus souvent le PèreLachaise - qui au fil des ans, épouse un parcours politique signifiant, avec ses éventuelles stations symboliques. Le 5 juin 1832, le cortège du général Lamarque, décrit par
2 novembre, les tombes d'opposants notoires voient converger des foules qui entrent là par effraction, dans la sphère publique et politique. Ainsi, le 2 novembre 1831, de nombreuses stations sont-elles observées auprès des tombeaux de Benjamin Constant et du député Manuel. Des graffitis politiques font aussi leur apparition sur certaines tombes, sans contrevenir au respect dû au défunt. Ainsi les sépultures du maréchal Ney et du général Labédoyère, victimes de la répression politique de 1815 , sont-elles ornées de tels graffitis appelant à la vengeance collective.

Le rite de l'éloge sur la tombe autorise aussi la politisation d'une pratique funéraire nouvelle. L'éloge profane est apparu dans les cimetières, sous le Directoire et s'y adjoint, sous la Restauration, un discours politique en surimpression. Évocations plus ou moins discrètes de la Révolution française et de la gloire napoléonienne, appels à la nation tout entière, avertissements politiques au régime en place: tels sont les passages obligés de ces éloges qui, autant qu'un défunt, célèbrent un passé enfoui et un futur espéré.

Le culte rendu aux "victimes politiques ", insurgées tombées aux combats, condamnées à mort politiques, passe par des voies plus tortueuses, mais participe également à l'apprentissage du politique sous les monarchies censitaires. Sauf exception, point ici de concession perpétuelle et de monument grandiose. Les condamnés à mort sont, d'après le Code pénal du 1810 , privés de toute sépulture individualisée, réduits à une inhumation "sans aucun appareil» (article 14). Mais une tradition orale peut perpétuer dans les mémoires la localisation supposée des suppliciés et autoriser des cultes clandestins. Ainsi la sépulture supposée des conspirateurs républicains de 1816 et celle des sergents de La Rochelle exécutés en 1822, sont-elles ornées en 1830 d'un drapeau tricolore. De même, la tombe pourtant dissimulée du maréchal Ney au Père-Lachaise fait l'objet de pèlerinages répétés, formés en grande partie de femmes venues déposer couronnes funèbres et feuilles volantes. Deux d'entre elles seront même arrêtées par les autorités en 1816.

Quant aux insurgés abattus lors des diverses «guerres de barricades» qui jalonnent la période (1827-1830-18321834-1839), ils sont bien souvent exposés derrière les vitrines macabres de la morgue, nus et éclairés afin d'être identifiés par des proches. L'exposition de cadavres anonymes est la règle à Paris depuis la création de la morgue en 1804, mais elle devient, dans les moments insurrectionnels, un rituel à forte charge politique. Les foules accourues au spectacle de la morgue viennent par 
leur geste rendre hommage aux «vaincus» tombés sur les barricades, autant que satisfaire un voyeurisme morbide. En juin 1832, Henri Heine constate ainsi qu' " on y faisait la queue comme au grand Opéra quand on donne Robert le Diable ${ }^{\natural}$.

Faute de corps, un lieu de mémoire peut faire office de cénotaphe et catalyser les émotions du deuil. Il en est ainsi de la colonne Vendôme avant le retour des cendres de Napoléon. À partir de 1830, lorsque le tabou napoléonien commence à s'estomper, l'anniversaire de la mort du héros, chaque 5 mai, est célébré par des pèlerinages ad hoc, avec dépôt de couronnes, d'éloges funèbres et de statuettes. Le rite est quelquefois réprimé par les autorités, en particulier en mai 1832, avant que la mort du roi de Rome ne vienne atténuer la crainte d'une restauration napoléonienne.

Les luttes politiques particulièrement vives de l'âge romantique ont trouvé à s'incarner puissamment dans le culte des morts. Chaque camp célèbre ses héros, ses grands hommes et ses martyrs, avec ses propres rites et ses propres traditions. La révolution funéraire du tournant du siècle, avec ses cortèges funéraires, ses grandes nécropoles, ses monuments somptueux, ses couronnes funèbres, a rendu possible un certain nombre de gestes politiques interdits dans l'espace public ordinaire. Du côté des pouvoirs, c'est une redéfinition de la légitimité et de la sacralité politiques qu'a autorisée le culte public des morts. Plus généralement, les hommages funèbres ont diffusé dans le corps social, au-delà du corps politique légal, des émotions et des sentiments qui n'ont pas peu contribué à la construction des principales cultures politiques contemporaines - légitimiste, libérale, bonapartiste, républicaine. Un modèle parisien de la mort en politique a bel et bien émergé à l'âge romantique: l'apprentissage de la représentation par le culte des morts, érigé en langage politique.

\section{BIBLIOGRAPHIE}

AGULHON, M. (1978). "La statuomanie et l'histoire », Ethnologie française, $\mathrm{n}^{\mathrm{os}} 2-3$, p. 143-172.

BEN AMOS, A. (2000). Funerals, Politics and Memory in Modern France, 1789-1996, Oxford, Oxford University Press.

BERTRAND, R. (1988). "La législation des sépultures », dans M. PERONNET, dir., Chaptal, Toulouse, Privat, p. 177-190.

BOUREAU, A. (1988). Le simple corps $d u$ roi. L'impossible sacralité des souverains français. $X V^{e}-X V I I I^{e}$ siècles, Paris, Les Éditions de Paris.

BOUTRY, P. (1990). "Le roi martyr. La cause de Louis XVI devant la cour de Rome (1820) », Revue de l'histoire de l'Église de France, LXXVI, 1990/1, p. 51-71.

BOWMAN, F.P. (1973). Le Christ romantique, Genève, Droz.

DE BAECQUE, A. (1997). La gloire et l'effroi. Sept morts sous la Terreur, Paris, Grasset.

DEWERPE, A. (2006). Charonne 8 février 1962. Anthropologie historique d'un massacre d'État, Paris, Gallimard, Folio histoire. [Troisième partie, «Sortir du meurtre»].

DURKHEIM, E. (1985, $1^{\text {re }}$ éd. 1908). Les formes élémentaires de la vie religieuse. Le système totémique en Australie, Paris, PUF.

FUREIX, E. (2002). «Un rituel d'opposition sous la Restauration : les funérailles libérales à Paris (1820-1830) », Genèses. Sciences sociales et histoire, $\mathrm{n}^{\circ} 46$, p. 77-100.

FUREIX, E. (2003). Mort et politique à paris sous les monarchies censitaires: mises en scène, cultes, affrontements, thèse de doctorat d'histoire sous la dir. d'A. Corbin, Université de Paris I (à paraître chez Champvallon).

FUREIX, E. (2004). «Banquets et enterrements », dans J.-J. BECKER et G. CANDAR, dir., Histoire des gauches, Paris, La Découverte, t. I, p. 197-209.

FUREIX, E. (2005). «Le deuil de la Révolution dans le Paris de la Restauration », dans J.-Y. MOLLIER, M. REID et J.-C. YON, dir., Repenser la Restauration, Paris, Nouveau Monde Éditions, p. 17-29.

GIESEY, R. (1987). Le roi ne meurt jamais. Les obsèques royales dans la France de la Renaissance, Paris, Flammarion.

GOFFMANN, E. (1991). Les cadres de l'expérience, Paris, Éditions de Minuit.

HINTERMEYER, P. (1981). Politiques de la mort tirées du concours de l'Institut. Germinal an VIII- Vendémiaire an IX, Paris, Payot.

JULLIARD, J. (dir.) (1999). La mort du roi. Essai d'ethnographie politique comparée, Paris, Gallimard.

LASSERE, M. (1997a). Villes et cimetières en France de l'Ancien Régime à nos jours: le territoire des morts, Paris, L'Harmattan.

LASSERE, M. (1997b). "Le XIX ${ }^{\mathrm{e}}$ siècle et l'invention du tourisme funéraire ", Revue d'histoire moderne et contemporaine, vol. 44, $\mathrm{n}^{\circ} 4$, p. 601-616.
OTTO, R. (1929). Le sacré. L'élément nonrationnel dans l'idée du divin et sa relation avec le rationnel, Paris, Payot.

OZOUF, M. (1984). «Le Panthéon», dans P. NORA, dir., Les Lieux de mémoire. La République, Paris, Gallimard, p. 139-166.

REDDY, W.M. (2001). The Navigation of Feelings. A Framework for the History of Emotions, Cambridge, Cambridge University Press.

TARTAKOWSKY, D. (1999). Nous irons chanter sur vos tombes. Le Père-Lachaise. $X I X^{e}-X X^{e}$ siècles, Paris, Aubier.

TILLY, C. (1984). «Les origines du répertoire de l'action collective contemporaine en France et en Grande-Bretagne », Vingtième siècle, $\mathrm{n}^{\circ}$ 4, p. 89-108.

URBAIN, J.-D. (1978). La société de conservation. Étude sémiologique des cimetières d'Occident, Paris, Payot.

VINCENT-BUFFAULT, A. (1986). Histoire des larmes, Paris, Rivages.

WAHNICH, S. (2002). «De l'économie émotive de la Terreur", Annales. Histoire, sciences sociales, vol. $57, \mathrm{n}^{\circ} 4$, p. 889-914.

\section{Notes}

1. Maurice Barrès, "La vertu sociale d'un cadavre », in Les déracinés, Paris, Gallimard, Folio, 1988 [1 $1^{\text {re }}$ éd. 1897], p. 465-466 (à propos des funérailles de Victor Hugo).

2. Carl Schmitt, La notion de politique. Théorie du partisan, Paris, Flammarion, 1992 [1 $1^{\text {re }}$ éd. 1963], p. 64.

3. Article "émotion» du Dictionnaire de la langue française de Laveaux (Paris, Ledentu, 1823): "Mouvements dans les humeurs, dans les esprits, dans l'âme. [...] Mouvement populaire qui précède les émeutes, les soulèvements. »

4. Maine de Biran, Journal, Neuchâtel, Éditions de la Baconnière, 1954-1957, t. 1, p. 39 (21 janvier 1815).

5. Ibid., p. 91 (21 juin 1815).

6. Edgar Quinet, Lettres à sa mère, t. 2, 18211825, Paris, Honoré Champion, 1998, p. 250 (12 décembre 1825).

7. Le Globe, 14 décembre 1830.

8. Le Moniteur universel, 21 janvier 1815.

9. Heinrich Heine, De la France. Traduction, notes et postface par Jean-Louis Besson, Paris, Cerf, 1996, p. 157. 